\title{
Scientific visualization to study Flux Transfer Events at the Community Coordinated Modeling Center
}

\author{
Lutz Rastätter* \\ Space Weather Laboratory, Mail Code 674, NASA GSFC, Greenbelt, MD 20771, USA \\ (Ph. +1 301286 1085, Fax: +1 301286 1648) \\ Maria. M. Kuznetsova \\ Space Weather Laboratory, Mail Code 674, NASA GSFC, Greenbelt, MD 20771, USA \\ (Ph.: +1 301286 9571, Fax: +1 301286 1648) \\ David. G. Sibeck \\ Space Weather Laboratory, Mail Code 674, NASA GSFC, Greenbelt, MD 20771, USA \\ (Ph.: +1 301286 5998, Fax: +1 301286 1648) \\ David H. Berrios \\ Science Data Processing Branch, Mail Code 587, NASA GSFC, Greenbelt, MD 20771, \\ USA (Ph.: 301286 9488, Fax: +1 301286 1648)
}

\begin{abstract}
In this paper we present results of modeling of reconnection at the dayside magnetopause with subsequent development of flux transfer event signatures. The tools used include new methods that have been added to the suite of visualization methods that are used at the Community Coordinated Modeling Center (CCMC). Flux transfer events result from localized recon-
\end{abstract}

\footnotetext{
${ }^{*}$ Lutz Rastätter

Email addresses: lutz.rastaetter@nasa.gov (Lutz Rastätter), maria.m.kuznetsova@nasa.gov (Maria. M. Kuznetsova), david.g.sibeck@nasa.gov (David. G. Sibeck), david.h.berrios@nasa.gov (David H. Berrios)

$U R L:$ http://ccmc.gsfc.nasa.gov (Lutz Rastätter)
} 
nection that connect magnetosheath magnetic field and plasma with magnetospheric fields and plasma and results in flux rope structures that span the dayside magnetopause. The onset of flux rope formation and the threedimensional structure of flux ropes are studied as they have been modeled by high-resolution magnetohydrodynamic simulations of the dayside magnetosphere of the Earth. We show that flux transfer events are complex three-dimensional structures that require modern visualization and analysis techniques. Two suites of visualization methods are presented and we demonstrate the usefulness of those methods through the CCMC web site to the general science user.

Keywords: scientific visualization, flux transfer events, magnetopause, magnetic reconnection

\section{Introduction}

Magnetohydrodynamic simulations are an important tool to obtain a global view of conditions in the Earth's magnetosphere and to make sense of spacecraft observations. Dayside reconnection in particular is an important process to transfer energy and momentum from the solar wind plasma in the magnetosheath into the magnetosphere As magnetic field lines reconnect on the dayside, plasma from the magnetosheath is transferred immediately into the high-latitude magnetosphere.

Dungey (1961) was the first to propose dayside reconnection as a mechanism to transfer energy from the solar wind into the magnetosphere. He imagined the reconnection process to occur in steady-state during southward interplanetary magnetic field (IMF) orientation. Closed magnetic field lines 
near the nose of the magnetosphere reconnect with solar wind field lines and the interconnecting field lines get dragged over the poles and down-tail to eventually reconnect back at nightside neutral points.

Satellite observations modified that picture leading to a multitude of models being proposed to describe reconnection events in the dayside. Russell and Elphic (1979) studied ISEE satellite data and found that reconnection occurs intermittently. They introduced the term Flux Transfer Event (FTE) and explained the phenomenon as an isolated flux tube filled with mixed plasma from the magnetosheath and magnetosphere. Disturbances caused by the FTE travel past an observer as the flux tube travels past the observing satellite. The flux tubes in the observations were characterized by a strong core magnetic field and a bi-polar signature during their passage relative to the satellite.

In general, anti-parallel reconnection normal to the nominal magnetosphere might be expected to create a weak core field (Ding et al., 1991) whereas a guide field may become amplified into a strong core field (Scholer, 1998).

A theoretical model of a flux rope structure was constructed using analytic functions by Hesse et al. (1990). Field line tracings through the analytic structure exhibit a mix of all the possible connections (magnetosheath field lines not connected to Earth, field lines connected to either the southern or northern hemisphere of the Earth, and closed field lines) within a small spatial region of the flux tube. Flux tubes found on the magnetopause move under the influence of magnetic curvature and pressure gradient forces. Satellites outside a nearby flux tube may observe transient enhancements of 
magnetic field strength and bipolar magnetic perturbations normal to the magnetopause surface. Satellites that pass through an FTE may see craterlike weakening of the magnetic field when there is no guide field in the reconnection region or core field enhancements with guide field (Sibeck et al., 2008; Kuznetsova et al., 2009).

Magnetic reconnection on the dayside magnetopause is most likely if there is a southward component of the IMF. However, a sizable east-west component $\left(B_{y}\right)$ may exist leading to component reconnection in the sub-solar region at the magnetopause. In this reconnection type a guide field remains at the diffusion region and opposing magnetic field components are confined to the plane perpendicular to that component. The reconnection current sheet is expected to follow at an angle half-way between the directions of the magnetosheath field and the (northward) magnetosphere field Sonnerup (1974). If reconnection occurs simultaneously along the line, the resulting flux tube is expected to be obliquely oriented (Lee and Fu, 1985; Sibeck et al., 2008).

Recent observations with CLUSTER and THEMIS (Lui et al., 2008) have shown that flux tube signatures vary with solar wind conditions and that satellites located close to each other may observe vastly different signatures as an FTE passes by. Sibeck et al. (2008) described the characteristics of an FTE that is embedded in a weakened magnetic field along the magnetopause current sheet that itself has a enhanced core field. In addition, the structure extends out into both the magnetosphere and magnetosheath. Kuznetsova et al. (2009) reported on the analysis of the FTE in numerical simulations that is the basis of this paper. A more detailed analysis has been conducted of the same event by Kuznetsova et al. (2011) by using the same simulation 
data

Simulations and visualizations of the magnetosphere are valuable tools to put those observations into context. In this paper we demonstrate the results of numerical simulations for selected events when FTEs have been observed and describe their shape from the simulations and similarities with observed signatures.

Here we demonstrate how the use of modern visualization tools can assist in the analysis of the internal structure of a modeled FTE and its internal mixing of magnetosphere and magnetosheath plasma by proxy of the magnetic field line connectivity. The tools allow us to track magnetic field lines within the flux tube and their connection into the Earth's ionosphere.

\section{Event and Magnetospheric Modeling}

The event that was modeled was a quasi-steady solar wind observed during May 20, 2007 shown in Fig. 1. The solar wind density ranged between 1 and 2 $\mathrm{cm}^{-3}$, the interplanetary magnetic field was duskward and southward (GSM $\left.B_{\mathrm{X}}, B_{\mathrm{Y}}, B_{\mathrm{Z}}=[0,3,-1] \mathrm{nT}\right)$ and the solar wind velocity was $V_{\mathrm{X}} \sim 550 \mathrm{~km} / \mathrm{s}$.

The model used was the Block-Adaptive Solar Wind Roe Upwind Scheme (BATS-R-US) (Powell et al., 1999) that was set to resolve the magnetopause on the dayside and flanks with cells of $1 / 16 R_{\mathrm{E}}$ size. The ionospheric Pedersen and Hall conductances were held constant at 5 and 0 mho, respectively. The simulation was published on the CCMC web site as magnetosphere run "David_Sibeck_112707_1".

Figure 2 shows the near-Earth magnetosphere in a noon-midnight meridional plane $(Y=0)$. Current density is shown in color and magnetic field 
lines emanating from selected position in the cut plane (shown projected onto the plane) as lines. Closed field lines are shown in red, magnetosheath and solar wind field lines in blue and open field lines in green (connected to northern hemisphere) and yellow (connected to southern hemisphere).

In this particular simulation, the inner magnetosphere $\left(=38 R_{\mathrm{E}}<X<\right.$ $\left.18 R_{\mathrm{E}},-12 R_{\mathrm{E}}<Y, Z<12 R_{\mathrm{E}}\right)$ is resolved at $1 / 8 R_{\mathrm{E}}$ and the dayside magnetopause and magnetosheath region $\left(X>-4 R_{\mathrm{E}}, 5 R_{\mathrm{E}}<\sqrt{(} X^{2}+Y^{2}+Z^{2}\right)<$ $\left.15 R_{\mathrm{E}}\right)$ is resolved at $1 / 16 R_{\mathrm{E}}$.

\section{Visualization suites and recent additions}

\subsection{Web visualization - server-side processing and rendering}

The primary mode of visualization is a server-side rendering system driven by a Perl interface and Interactive Data Language (IDL ${ }^{\circledR}$ by ITT Visualization Solutions) program collection. The IDL script written by the interface reads the model output file with the three-dimensional data and renders an image to the user's specifications. This system runs in batch mode on the CCMC web server and usually returns an image within a minute directly into the user's web browser. This system has been in place at CCMC since 2000 and now supports over 20 different models from the solar corona, heliosphere, magnetosphere to the Earth's ionosphere and 30 output formats.

In this paper we demonstrate new features that are applicable to simulations of the Earth's magnetosphere. To view results of the above mentioned simulation on the CCMC web site (http://ccmc.gsfc.nasa.gov), one selects "View Results" on in the navigation bar, then go to "Global Magnetosphere Model" then search for the run name, e.g., "David_Sibeck_112707_1". 
The individual run's web page then presents the user with basic information about the simulation run with links to the solar wind data (plot image), links to the visualization of the magnetosphere and ionosphere outputs and, if requested, model outputs along satellites flown through the magnetosphere during the simulation. In newer simulations (starting in 2011) all applicable satellites in the magnetosphere are flown through the model output data (at the requested output cadence of between 1 and 10 minutes) regardless of whether they have been requested. Satellites requested during the run submission will have a higher time cadence (typically a record is written every 5 to 15 seconds).

\subsubsection{Field line topology}

The topology of magnetic field lines is determined from 3D magnetospheric model outputs by tracing the magnetic field from positions on the plot slice. Doing this for each position on a grid may involve tracing many thousands of field lines and is computationally expensive. We employ an adaptive method that starts from a very coarse base grid in the cut plane (between 5 and 11 positions in each direction). After field lines have been computed for each position on the base grid, the topology values of the field lines are noted on each grid vertex. Different topology values are assigned for (1) a field line not connected to Earth, (2) a closed field line connected to Earth at both ends, (3) an open field line connected only to the southern hemisphere and (4) only the northern hemisphere. In each successive refinement step, cells within the grid that have corners with varying topology values will be subdivided into four cells. As a cell is split, field lines emanating from the additional 5 vertices (half-way points on the edges and the center of the 
unrefined cell) are computed and topology results recorded. Up to 5 levels of refinement can be requested in the online interface to improve the resolution of the base grid by a factor of 32 in both directions in the cut plane. Each refinement step actually consist of two sweeps: 1) The first sweep makes sure that cells adjacent to refined cells are also subdivided if the topology varies along any of the edges or if the resolution varies by more then a factor of four (two adaptation levels). 2) The second sweep refines cells that have varying topologies at their corner vertices. This two-step process ensures that boundaries between different topologies are uniformly resolved.

Vertices that fall outside of the simulation domain or within the inner boundary of the magnetospheric model solution, typically $3 R_{\mathrm{E}}$, are assigned a missing value. Cells that have a vertex with missing topology that differs from the other topologies are not refined unless the cell's center is located within $10 R_{\mathrm{E}}$ of the Earth. This is done to resolve the inner magnetosphere and cusp area.

\subsection{Java ${ }^{\circledR}$ Web Start application Space Weather Explorer}

The Space Weather Explorer (SWX), version 2, is a stand-alone Java ${ }^{\circledR}$ based tool that runs on a user's computer. This tool is downloaded from the CCMC web page (under Downloads and SWX-2) and started automatically via the locally installed Java Web Start (JavaWS) application. SWX requires version 6 or newer of Oracle's Java Runtime Environment (JRE) libraries to run on the user's computer and requires a model output file (written by CCMC's Kameleon converter) on the local hard disk. To obtain the SWX application from the CCMC web site via the "Downloads" link in the upper row, select "Space Weather Explorer" and navigate to the web page for SWX- 
2 (http://ccmc.gsfc.nasa.gov/swx2/).

Sample model output files are available on the SWX version 2 web page, including data from the "David_Sibeck_112707_1" run that was used in this paper. Requests for output files of other simulation runs can be made on the CCMC website. The SWX page has a link to the "CDF Request Form" which allows users to specify model output files needed. Individually prepared files are provided to the user via the CCMC FTP server (download links are sent by email).

The Java application allows for more interactive visualization including instant rotation of the view angle and addition of traced flow lines or field lines to the plot. Version 2 of the Space Weather Explorer is undergoing active development. Recent functionality updates include the ability to render multiple cut slices together with field lines that can be added by mouse clicks and by importing lists of start positions. We use this "Import" feature to read in lists of field line start positions. Different versions of SWX are listed on the page in case the current version does not work as well as older versions for a particular purpose.

\section{Results}

\subsection{Magnetic topology}

The topology of the magnetic field can now be rendered online at sufficient spatial resolution using the adaptive algorithm.

Figure 3 shows the magnetic topology in the meridional $(Y=0)$ cut plane for the same area as in Fig. 2 for (a) 20:41:20 UT and 22:14:20 UT in the simulation. The topology was computed on a base grid of 11x6 positions 
then refined four times to obtain a spatial resolution of about $3 / 16 R_{\mathrm{E}}$.

The resulting 321x161 positions required 4947 field lines (7880 for the later time 22:14:20 due to the much more complicated structures in the magnetotail) with the adaptive method as opposed to 51681 field lines when using the conventional method of checking every grid position. Using different numbers of adaptation levels, it is found that for typical magnetospheric cut slices, the number of field lines traced increases by a factor of about 2.5 with each added adaptation level, well below the factor of 4 needed with the conventional method.

The initial base grid is essential to detect the global structure in which to start the refinement properly and to find all the edges between regions of different topology. Between 5 and 11 positions in each direction is found to be optimal and the number of refinements should be between 3 and 5 to render an image with suitable resolution.

A flux tube of a Flux Transfer Event can be a complicated three-dimensional structure as can be seen in Figure 4, which shows a series of cuts through an FTE flux tube. Panels (a) show the magnetic field strength $B$ at $Y=-2 R_{\mathrm{E}}$, $Y=0 R_{\mathrm{E}}$, and $Y=+2 R_{\mathrm{E}}$ similar to Figure 4 in Kuznetsova et al. (2011). At $Y=0$ on the magnetopause the structure shows a pronounced round enhancement of $B$, whereas the adjacent cuts show a weaker signature and a flatter structure. Panel (b) shows the magnetic topology in the same vertical cuts as Panel (a). In the center plot at $Y=0$ we see regions with mostly green (connected to southern hemisphere) at the top, yellow (connected to northern hemisphere) at the bottom, some blue (magnetosheath) in the center of the flux tube and red (closed) on the surface for the flux 
rope. The distribution of the field line topologies is quite different in the other cut planes: At $Y=-2$ green is at the bottom and yellow at the top. At $Y=2$ yellow is mostly in the center and green wrapped around together with red. This shows that the flux tube is tightly twisted, a fact that will be demonstrated by SWX in the next section.

Figure 5 shows the adaptive determination of magnetic topology in a the same area as shown in Panel (b) of Figure 4. Panel (a) shows the base grid and one level of refinement, Panel (b) shows two additional levels of refinement and the FTE structure that is starting to take shape. Panel (c) has all 5 refinement levels and the FTE structure is resolved at $1 / 32 R_{\mathrm{E}}$ The final plot (in Figure 4)is created by interpolating the topology values assigned to the vertices of the adaptive grid to an equidistant grid with the finest resolution of the adaptive grid. Coarser cells with uniform topology values have been oversampled to be plotted at the finest resolution.

We will use this topology information to define field line start positions in the following section.

\subsection{Three-dimensional flux rope}

Figure 6 (a) shows the field line start positions relative to the strength of the current density $J$ obtained with the $\mathrm{CCMC}$ online visualization tool. The two-dimensional grid of start positions samples the area covered by the FTE and the surrounding magnetosphere and magnetosheath. Positions inside

the FTE are roughly located within an elliptical shape $(+$ signs $)$. Field lines resulting from the inside region are rendered in panels (c) and (d). The three-dimensional visualization shows a tightly wound flux tube with the intertwined field lines with topologies indicated by red (closed), white 
(open) and yellow (magnetosheath).

Field lines that thread the southern magnetosheath on the afternoon side are connected to the flux tube and connect to the Earth (white lines) or wrap around and remain unconnected (yellow field lines). Closed field lines connect the afternoon side of the northern hemisphere with the morning side in the southern hemisphere. In the southern hemisphere, field line foot points of closed and open field lines that are connected to the flux tube all map closely together near the lower-latitude boundary of the field-aligned current pattern at the model's inner boundary (spheres of $3.1 R_{\mathrm{E}}$ in panels (c) through (f). In the northern hemisphere, field line foot points are split between two positions near the cusp near local noon (about 13 hours local time) and further in the afternoon side (about 14 hours of local time). The foot points near local noon are shared with closed field lines from the periphery of the FTE seen in panels (e) and (f). Open field lines emanating from the patch of foot points at local noon in the northern hemisphere thread the flux rope and diverge from it in the morning side, along with magnetosheath field lines that thread the entire flux tube from afternoon side to morning side. Open field lines connected to the southern hemisphere diverge from the flux rope all along the afternoon side. Closed and open field lines that reach the inner boundary at 14 hours local time in the northern hemisphere thread the flux rope all the way to about $Y=+9 R_{\mathrm{E}}$ where the flux tube ends.

The three-dimensional visualization tool shows how magnetosheath and solar wind field lines (shown in yellow) are intertwined with partially open field lines (connected to one hemisphere of the Earth, grey) and closed field lines (red). At this time, reconnection occurs at the flanks of the magneto- 
sphere (to the left and right of the $Y-0$ plane where the yellow (magnetosheath) field lines are kinked. On the right side more evidence of reconnection are the field lines that connect to the Earth (red) and open field lines (grey) that diverge at one point from the flux tube.

The reconnection that leads to the formation of FTEs and the FTEs structure can be studied in great detail with SWX in addition to the twodimensional online visualization available at CCMC.

\section{Summary and Conclusions}

Magnetic flux transfer events (FTEs) are complicated three-dimensional structures that cannot be described by two-dimensional approximations. The characteristics of the flux tube cross section can vary dramatically within a few Earth radii from a given cut plane where satellites may observe it. Reconnection responsible for the formation of those flux ropes may occur far away in the flanks of the magnetosphere. Resulting field lines are highly intertwined and plasma populations from the magnetosphere, magnetosheath and solar wind can be found in close proximity.

We have demonstrated the power of scientific visualization methods that are available at the Community Coordinated Modeling Center. The new visualization of magnetic field topology that is obtained by tracing magnetic field lines throughout the three-dimensional magnetosphere is a complement to the tho-dimensional visualization that shows scalar and vector variables, that is currently available on the $\mathrm{CCMC}$ website. An adaptive start point grid reduces the large amount of field line tracings needed and makes it feasible to visualize the magnetic topology in high resolution for large simulations 
(up to 20 million grid cells in a BATS-R-US simulation) through the CCMC visualization web interface.

A separate tool developed for client-side analysis, the Space Weather Explorer, supports interactive selection of field line start points and real-time manipulation of a plotted scene. The Space Weather Explorer is a useful tool not only for scientific analysis but serves educational purposes by familiarizing a user with the three-dimensional structure of the Earth's magnetosphere and of the interaction with the solar wind.

Currently, we are developing a JavaScript interface that will operate the Space Weather Explorer on the web server side. We hope that in the near future the interaction speed becomes comparable to the speed of the downloaded SWX application reading a data file on the user's computer. This can be achieved by sending requests to the server and receiving results as small data packets that are rendered directly within the web browser. We are working on implementing the same functionality, including selection of field line start positions and multiple cut planes. Available simulation runs and time steps would be selected and analyzed immediately without the need to request and download large data sets. This will make the visualization process much more efficient.

\section{Acknowledgements}

This work was funded as part of the Community Coordinated Modeling Center's operation. Work by D. G. Sibeck and M. M. Kuznetsova was supported in part by funding from the NASA Heliophysics Guest Investigator Program. 


\section{References}

Ding, D.-Q., Lee, C., Ma, Z.-W., 1991. Different FTE signatures generated by the bursty single $\mathrm{X}$ line reconnection and the multiple $\mathrm{X}$ line reconnection at the dayside magnetopause. Journal of Geophysical Research 96, $57 @$ S66.

Dungey, J. W., 1961. Interplanetary magnetic field and the auroral zones. Physical Review Letters 6, 47.

Hesse, M., Birn, J., Schindler, K., MAY 1 1990. On the Topology of Flux Transfer Events. Journal of Geophysical Research 95 (A5), 6549-6560.

Kuznetsova, M. M., Sibeck, D. G., Hesse, M., Rastaetter, L., Berrios, D. H., nd, G. T., Gombosi, T. I., 2011. Three-dimensional structure of Flux Transfer Events. Journal of Geophysical Research, submitted.

Kuznetsova, M. M., Sibeck, D. G., Hesse, M., Wang, Y., Rastaetter, L., Tóth, G., Ridley, A., MAY 22 2009. Cavities of weak magnetic field strength in the wake of FTEs: Results from global magnetospheric MHD simulations. Geophysical Research Letters 36.

Lee, L., Fu, Z., 1985. A Theory of Magnetic Flux Transfer at the Earth's Magnetopause. Geophysical Research Letters 12 (2), 105-108.

Lui, A. T. Y., Sibeck, D. G., Phan, T., Angelopoulos, V., McFadden, J., Carlson, C., Larson, D., Bonnell, J., Glassmeier, K.-H., Frey, S., 2008. Reconstruction of a magnetic flux rope from THEMIS observations. Geophysical Research Letters 35. 
Powell, K. G., Roe, P. L., Linde, T. J., Gombosi, T. I., De Zeeuw, D. L., 1999. A solution-adaptive upwind scheme for ideal magnetohydrodynamics. Journal of Computational Physics 154 (2), 284.

Russell, C., Elphic, R., 1979. ISEE Observations of Flux-Transfer Events at the Dayside Magnetopause. Geophysical Research Letters 6 (1), 33-36.

Scholer, M., 1998. Magnetic flux transfer at the magnetopause based on single X-line bursty reconnection. Geophysical Research Letters 15, 291.

Sibeck, D. G., Kuznetsova, M. M., Angelopoulos, V., Glaßmeier, K.-H., McFadden, J. P., 2008. Crater FTEs: Simulation results and THEMIS observations. Geophysical Research Letters 35.

Sonnerup, B. U. O., 1974. Magnetopause reconnection rate. J. Geophys. Res. 79, 1546-1549.

\section{Figures}




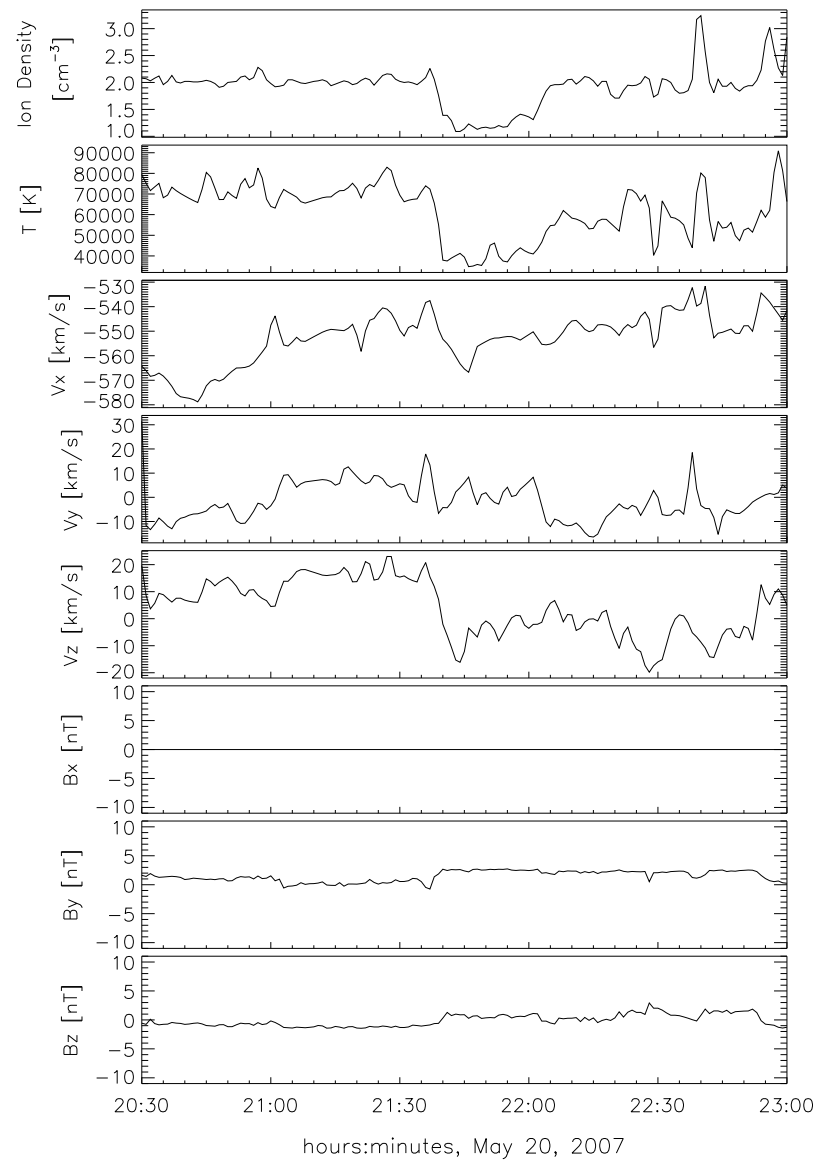

Figure 1: Solar wind as measured by ACE for May 20, 2007. 
a)

05/20/2007 Time $=20: 51: 20$ UT $y=0.00 R_{E}$

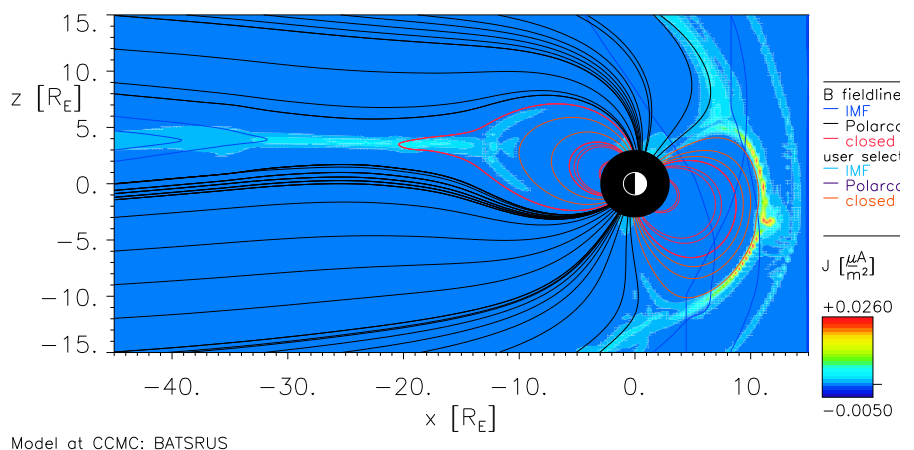

b) $\quad 05 / 20 / 2007$ Time $=22: 14: 20$ UT $y=0.00 R_{E}$

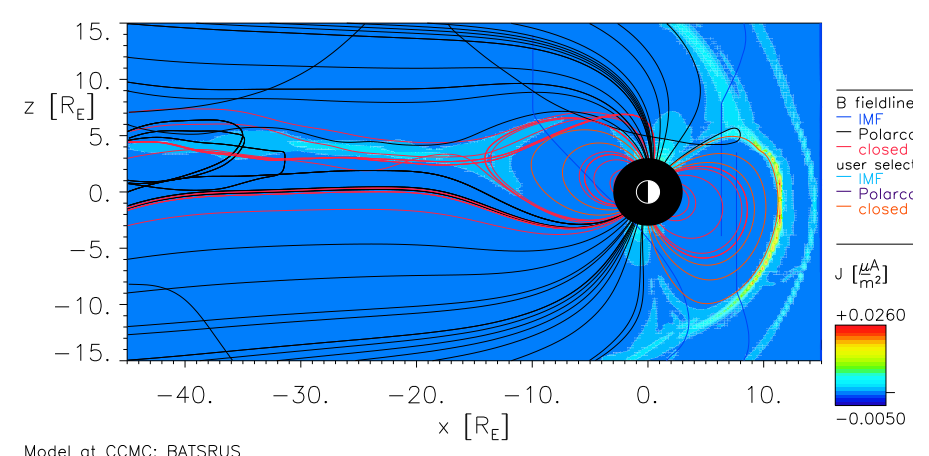

Model at CCMC: BATSRUS

Figure 2: Near-Earth magnetosphere at $\mathrm{Y}=0$ at (a) 20:51:20 UT and (b) 21 22:14:20 UT. The total current density is shown in color and magnetic field lines started from selected locations in the Y-0 plane as colored lines. The colors represent the magnetic field topology with red: closed, blue: solar wind or magnetosheath field lines, black: open field lines connected either to the northern $\$ 8$ the southern hemisphere of the Earth. 


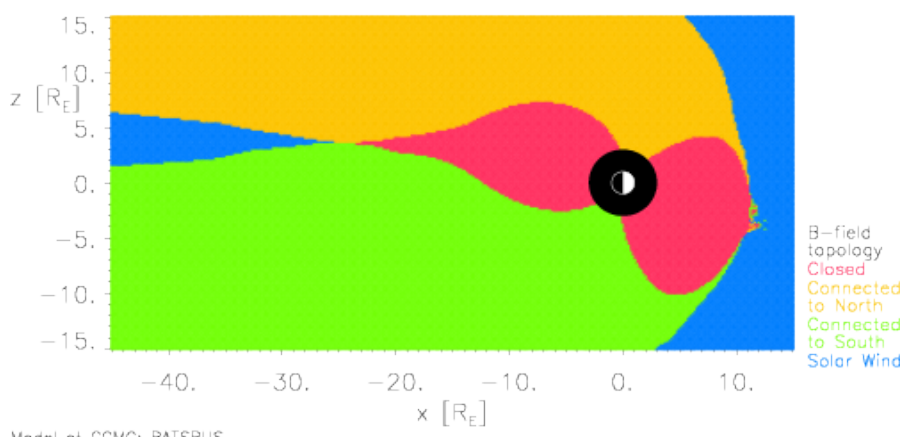

Modet of CCMC: BATSRUS

b)

$05 / 20 / 2007$ Time $=22: 14: 20$ UT $y=0.00 R_{E}$

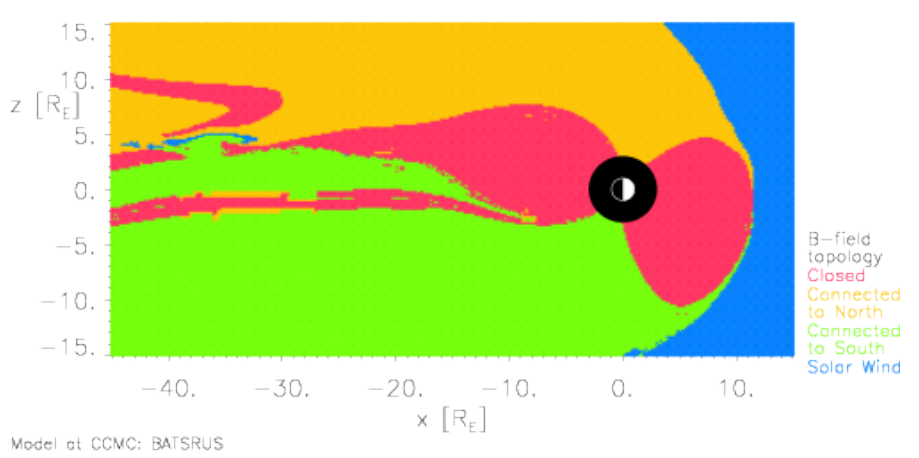

Model ot COMC. BATSRU

Figure 3: Near-Earth magnetosphere field topology at $Y=0$ at (a) 20:51:20 UT and (b) 22:14:20 UT. The colors represent the magnetic field topology. Red are closed field lines, blue: field lines of the solar wind or the magnetosheath (in agreement with field lines in Figure 2). Green and yellow are positions of open field lines connected either to the southern or northern hemisphere of thd $\mathrm{Warth}$, respectively. Panel (a) shows a Flux Transfer Event signature on the dayside (near the sub-solar point on the magnetopause (between the areas of red and blue), (b) a dynamic tail configuration. 

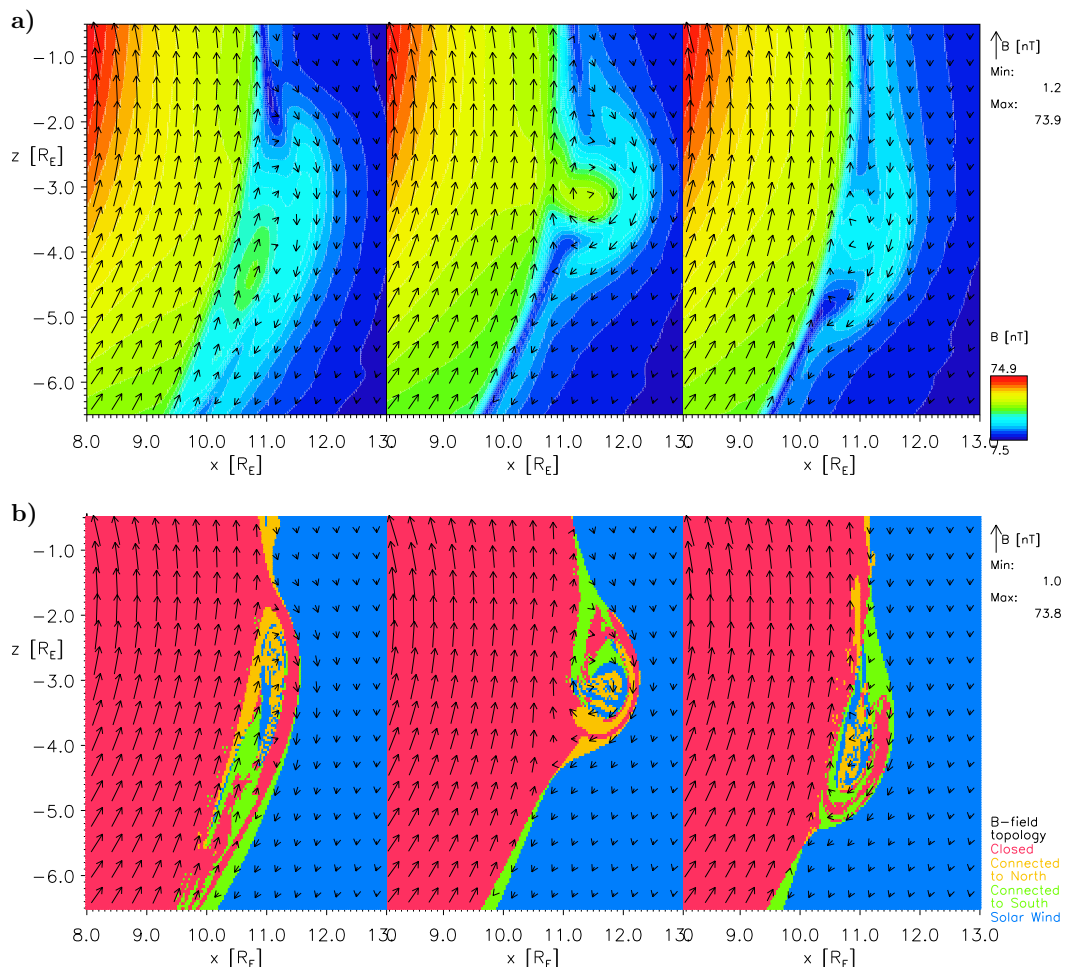

Figure 4: Cuts through the dayside magnetopause at 20:51:20 with a modeled FTE near the nose of the magnetosphere: Slices at left are at $Y=-2$, center at $Y=0$, and right at $\mathrm{Y}=2$ showing (a) magnetic field strength (B) in color and in-plane magnetic field (arrows), (b) magnetic field topology as color and in-=plane magnetic field as arrows. The color scheme for magnetic field topology is the same as in Figure 2. 

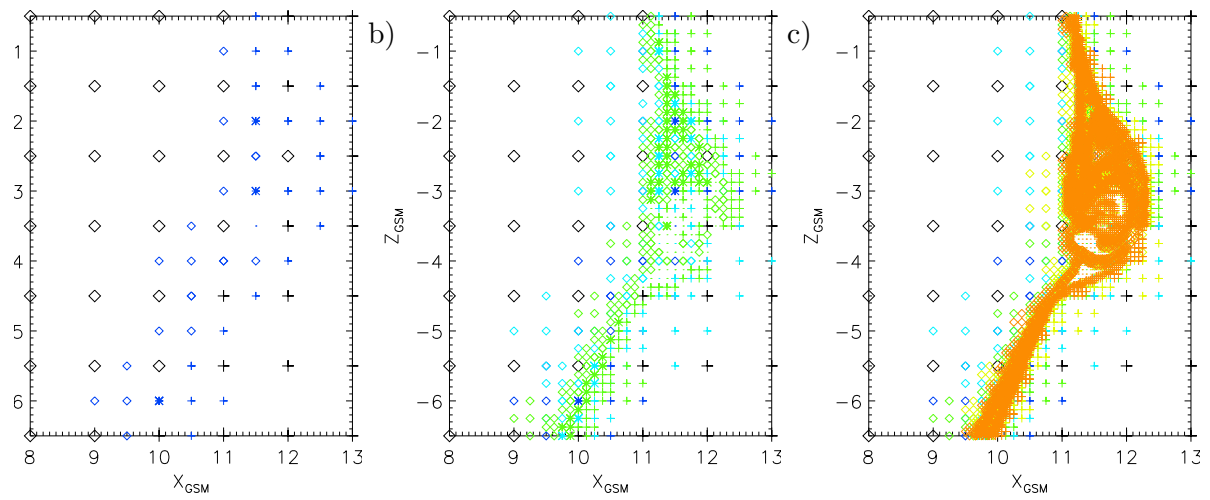

Figure 5: Adaptive grid used to compute field line topology for the center plot in Figure 4 (b). Panel (a) shows the 6x7-element base grid (black symbols) in the $5 R_{\mathrm{E}} \mathrm{x} 6 R_{\mathrm{E}}$ region and one level of refinement (blue). Panel (b) has two additional levels of refinement (magenta, green) and panel (c) includes 2 more refinements (yellow, orange) to complete the final set of 5 refinements. The 4 topology values in these plots are represented by different symbols: $\diamond$ : closed, $*$ : open, connected to south, $\cdot:$ connected to north, and + : magnetosheath field lines. 

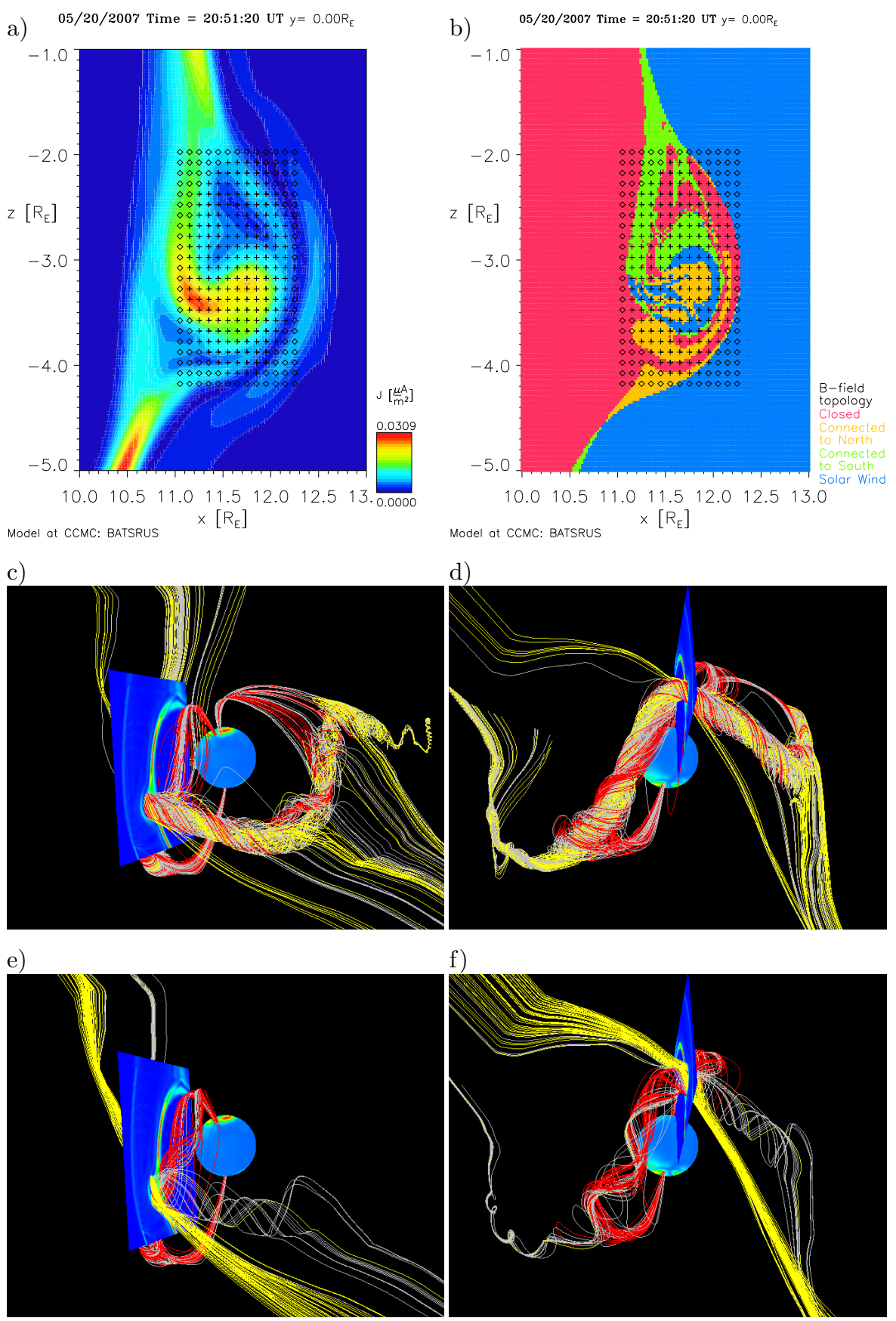

Figure 6: 3D field line tracings in and around an FTE. (a) FTE shown in magnetopause current density $J$ and (b) field line topology. Plus $(+)$ signs indicate field line start points within the FTE and diamonds $(\diamond)$ indicate 22 field lines outside the FTE. (c) SWX rendition of field lines from inside the FTE as seen from the afternoon side and (d) as seen from south of the direction of the Sun. The plane shown is the $Y=0$ plane with the current density as shown in (a). The sphere shows current densities at $R=3.1 R_{\mathrm{E}}$ around the Earth. Panels (e) and (f) show the field lines emanating from position outside the FTE, 